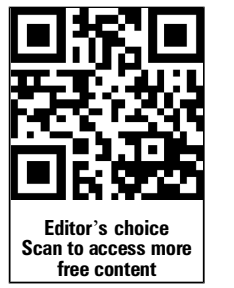

free content

\section{Handling editor Tore K Kvien \\ - Additional material is published online. To view please visit the journal (http:/l dx.doi.org/10.1136/ annrheumdis-2013-204715). \\ 1 Division of Clinical Immunology and Rheumatology, University of Alabama at Birmingham, Birmingham, Alabama, USA ${ }^{2}$ Department of Epidemiology, University of Alabama at Birmingham, Birmingham, Alabama, USA \\ ${ }^{3}$ Division of Preventive Medicine, University of Alabama at Birmingham Birmingham, Alabama, USA ${ }^{4}$ Health Sciences Research, Mayo Clinic, Rochester, Minnesota, USA}

\section{Correspondence to} Dr Jeffrey R Curtis, Division of Clinical Immunology and Rheumatology, University of Alabama at Birmingham, 510 20th Street South, Faculty Office Towers 802D, Birmingham, AL 35294, USA; jcurtis@uab.edu

Received 4 October 2013 Revised 21 January 2014 Accepted 16 February 2014 Published Online First 5 May 2014

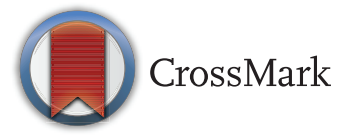

\section{Linked}

- http://dx.doi.org/10.1136/ annrheumdis-2013-204933

To cite: Zhang J, Chen L, Delzell $\mathrm{E}$, et al. Ann Rheum Dis 2014;73:1301-1308.

\title{
The association between inflammatory markers, serum lipids and the risk of cardiovascular events in patients with rheumatoid arthritis
}

\author{
Jie Zhang, ${ }^{1,2}$ Lang Chen, ${ }^{1}$ Elizabeth Delzell, ${ }^{2}$ Paul Muntner, ${ }^{2}$ William B Hillegass, ${ }^{3}$ \\ Monika M Safford, ${ }^{3}$ Iris Yolanda Navarro Millan, ${ }^{1}$ Cynthia S Crowson, ${ }^{4}$ \\ Jeffrey $R$ Curtis ${ }^{1,2}$
}

\section{ABSTRACT}

Objective To examine the association of serum inflammatory markers (erythrocyte sedimentation rate (ESR) and C-reactive protein (CRP)) and serum lipid measures (low-density lipoprotein (LDL)- and highdensity lipoprotein (HDL)-cholesterol) with risk of myocardial infarction (MI) and ischaemic stroke (IS) among rheumatoid arthritis (RA) patients.

Methods We conducted a retrospective cohort study using 2005-2010 data from a US commercial health plan. Eligible patients had two or more physician diagnoses of RA during a baseline period of at least 180 days with continuous medical and pharmacy coverage. We computed age-adjusted incidence rates of $\mathrm{MI}$ and $\mathrm{IS}$, and used spline regression to assess nonlinear associations and Cox-regression to quantify the independent association between the laboratory values and the outcomes.

Results We identified 44418 eligible RA patients (mean age 49 years; $76 \%$ women). CRP $>10 \mathrm{mg} / \mathrm{L}$ compared with $<1 \mathrm{mg} / \mathrm{L}$ was associated with increased $\mathrm{MI}$ risk (HR 2.12; 95\% Cl 1.02 to 4.38). ESR>42 mm/h compared with $<14 \mathrm{~mm} / \mathrm{h}$ was associated with increased risk of $\mathrm{MI}$ (HR 2.53; $95 \% \mathrm{Cl} 1.48$ to 4.31) and IS (HR $2.51 ; 95 \% \mathrm{Cl} 1.33$ to 4.75$)$ risk. HDL-cholesterol $\geq 60 \mathrm{mg} / \mathrm{dL}(1.6 \mathrm{mmol} / \mathrm{L})$ compared with $<40 \mathrm{mg} / \mathrm{dL}$ $(1.0 \mathrm{mmol} / \mathrm{L})$ was associated with reduced MI risk (HR $0.37 ; 0.21$ to 0.66 ). The association between LDL and MI was not linear; the lowest risk was observed among patients with $\mathrm{LDL}$ between $70 \mathrm{mg} / \mathrm{L}(1.8 \mathrm{mmol} / \mathrm{L})$ and $100 \mathrm{mg} / \mathrm{L}$ (2.6 mmol/L). We did not observe a significant association between LDL and IS.

Conclusions This study provides evidence supporting the hypothesis that RA-related systemic inflammation plays a role in determining cardiovascular risk and a complex relationship between LDL and cardiovascular risk.

\section{INTRODUCTION}

Patients with rheumatoid arthritis (RA) are at increased risk for cardiovascular disease (CVD) compared with those without RA. ${ }^{1}$ RA is associated with a twofold to threefold higher risk of myocardial infarction (MI) and a 50\% higher risk of stroke. $^{2}{ }^{3}$ The absolute risk of having a CVD event was examined in a cohort of recently diagnosed RA patients and age-matched and sex-matched non-RA individuals. $^{4}$ Among those 50-59 years of age, $83 \%$ of RA patients and $28 \%$ of non-RA individuals had $>10 \%$ risk of having a CVD event within 10 years, and the percentage increased to 100 among RA patients 60 or older. ${ }^{4}$ RA patients are also disproportionately affected by high CVD mortality. A meta-analysis of 111758 RA patients and 22927 CVD events reported increased CVD mortality in RA patients, with a standardised mortality ratio of $1.5(95 \%$ CI 1.4 to 1.6$) .^{5}$

The reasons for these excess risks are not well understood. While some traditional CVD risk factors have been shown to be more prevalent among RA patients compared with those without RA, such as smoking and elevated C-reactive protein (CRP) levels, they explain only a portion of the observed excess CVD risk in RA patients. ${ }^{3}{ }^{6}$ Framingham and Reynolds risk scores have been suggested to underestimate CVD event risk by 50\% among RA patients. ${ }^{8}$ Furthermore, a recent study reported that even when the modified EULAR systematic coronary risk evaluation (mSCORE) was used, a scoring system with a multiplier adapted for use in RA patients, $63 \%$ of RA patients at moderate cardiovascular risk were found to have carotid plaque on ultrasound. ${ }^{9}$

Studies to identify additional biomarkers and to further refine CVD risk prediction models for use in RA patients have been called for. ${ }^{10} \mathrm{~A}$ number of studies reported a positive association between markers of RA disease activity and CVD risk, suggesting that part of the excess CVD risk may be attributable to RA-related systemic inflammatory processes. ${ }^{11-13} \mathrm{CRP}$ and erythrocyte sedimentation rate (ESR) are important serum markers of RA disease activity. In the general population, CRP levels $>3 \mathrm{mg} / \mathrm{L}$ have been associated with increased CVD risk; in RA patients, one study reported a positive association between CRP and CVD risk that approached statistical significance (HR 1.10; 95\% CI 0.99 to 1.22$).{ }^{11}$ However, because up to $70 \%$ of RA patients may have CRP $>3 \mathrm{mg} / \mathrm{L},{ }^{14}$ the association between higher CRP levels and CVD risk is yet to be further elucidated. Furthermore, the cumulative inflammatory burden, measured by CRP and ESR levels measured over an extended period, has been associated with subclinical atherosclerosis, cardiovascular risk and mortality. ${ }^{15} \quad 16$ Elevated ESR has been associated with increased CVD risk in RA patients in two additional analyses. ${ }^{11} 17$ Yet there are little data comparing the two inflammatory measures with regard to their associations with cardiovascular risk in the same analysis. 
Further complicating the assessment of CVD risk among RA patients, the contribution of lipids is currently a matter of debate. ${ }^{17-19}$ Among RA patients, higher high-density lipoprotein (HDL)-cholesterol has been shown to be associated with reduced CVD risk in one retrospective cohort study ${ }^{17}$ and with increased CVD risk in another. ${ }^{11} \mathrm{~A}$ comparison of blood samples taken at least 10 years before the onset of RA to those from non-RA controls reported elevated levels of total cholesterol and triglyceride prior to RA onset among those who later developed RA than the controls. ${ }^{19}$ In contrast, other studies found lower levels of total cholesterol and triglyceride among RA compared with non-RA patients ${ }^{20}$ and a significant decline in these measures among RA patients prior to disease onset. ${ }^{21}$ In addition, one study reported an association between lower total cholesterol and lower low-density lipoprotein (LDL)-cholesterol with increased CVD risk, in contrast to that in the general population. ${ }^{11}$

Given these uncertainties, the objective of the current study was to examine the association between serum inflammatory markers (ESR and CRP) and lipid measures (LDL- and HDL-cholesterol) and risk of MI and ischaemic stroke (IS) in a large retrospective cohort study of RA patients enrolled in a US commercial health plan.

\section{METHODS}

\section{Study population}

Data for the current analyses were derived from the Optum Insight Database for the period 2005 to 2010, which contained longitudinal information on more than 90 million individuals since 1993. The database included claims for services provided at inpatient and outpatient settings and outpatient prescription drug experience among individuals enrolled in a nationwide private health plan.

Eligible participants for the current study were required to satisfy the following criteria: (1) had at least two RA diagnoses from physician encounters that were 7-365 days apart; (2) had at least 180 days of continuous coverage with medical and pharmacy benefits; and (3) had at least one of four laboratory tests (CRP, ESR, HDL- or LDL-cholesterol) performed either during baseline or follow-up with results recorded electronically. Follow-up started on the first day (index date) when criteria 1 and 2 were satisfied and stopped when the patients were hospitalised for MI or IS, died, lost health plan coverage or reached 31 December 2010. The 180 days prior to follow-up start was defined as the baseline period. RA patients who were hospitalised for MI or IS during the baseline period were excluded. If patients were censored due to loss of health plan coverage but subsequently re-enrolled, they could contribute additional follow-up time (additional 'episodes of care') but had to meet the eligibility criteria again.

\section{Identification of study outcomes}

The study outcomes were hospitalised MI and IS occurring during follow-up and were captured using claims-based algorithms that have been validated in prior studies to have high positive predictive values (PPVs). IS cases were identified by ICD-9-CM code 433.x1, 434 (excluding 434.x0) or 436 in any position on the discharge diagnosis list associated with inpatient hospitalisation at an acute care hospital (ie, not a rehabilitation hospital). MI cases were identified by code 410.x1 at any position associated with inpatient hospitalisations at an acute care facility and a minimal length of stay of two nights except for cases in which the patient died within two nights from admission. These algorithms have been associated with high PPVs to identify the respective outcomes: IS $(85-91 \%)^{22} 23$ and MI (86$97 \%) .^{24-26}$

\section{Main exposures and covariates of interest}

Laboratory data were obtained from tests ordered during routine care of a subset of patients who had their laboratory tests performed at laboratory networks, which record test results electronically. We identified these patients and linked their medical and pharmacy claims to their laboratory results for ESR, CRP, hs-CRP, HDL-cholesterol and LDL-cholesterol. We included laboratory values that occurred during the 6-month baseline period and during follow-up, but prior to an outcome event. Lab results were treated as time varying. In as much as CRP and ESR could be elevated as a consequence of an MI or stroke, and cholesterol might likewise be affected by an evolving ischaemic event, labs occurred within 14 days prior to the event dates were excluded to avoid measuring lab results too proximate to the outcome events of interest.

Age and gender were ascertained as of the study index date. Clinical characteristics were ascertained from the claims during the baseline period (for fixed covariates), which included comorbidities, diabetes diagnosis from a physician and hypertension diagnosis from a physician; and during baseline and follow-up for use of statins and non-statin lipid-lowering medications, and use of RA medications (biologics, non-biologic DMARDs and oral glucocorticoids). Comorbidities were measured using the Charlson Comorbidity Index, which has been adapted for use with administrative data and has been shown to predict short-term mortality and other serious outcomes. ${ }^{27}$ Exposure to statins, non-statin lipid-lowering medication, biologics, methotrexate and all other non-biologic DMARDs were captured as time varying on a person-day basis. Daily drug arrays were constructed in order to assess drug therapy based on fill dates and days of supply dispensed for each prescription.

\section{Statistical analysis}

We characterised the distribution of patient characteristics as mean (SD) or number (percentage). To allow for examination of non-linear effect between the biomarker levels and CVD risk, we used smoothing splines, ${ }^{28}$ adjusting for age and gender. We then computed the age-adjusted (to census 2010 data) incidence rates of hospitalised MI and stroke during follow-up stratified by gender and by biomarker levels. Cut-points from the Adult Treatment Panel (ATP) III guidelines were used to categorise the HDL-cholesterol and LDL-cholesterol values ${ }^{29}$; an additional cut-point for LDL-cholesterol was applied in the Cox regression analysis suggested by the spline. Clinically meaningful cut points for CRP $(<1,1-2,3-10$ and $>10 \mathrm{mg} / \mathrm{L})$ and ESR $(<14,14-27$, $28-42$ and $>42 \mathrm{~mm} / \mathrm{h}$ ) were applied. ${ }^{14}{ }^{30}$ Finally, we examined the associations between the inflammation and lipid markers with CVD risk using Cox regression adjusting for age, gender, comorbidities, use of lipid-lowering medications and use of RA medications. For the Cox regression analysis, we used a robust sandwich variance estimator to correct for correlated data because patients $(<5 \%)$ could contribute more than one episode to the analysis. ${ }^{31}$

\section{Sensitivity analysis}

Because the use of lipid-lowering medications and RA medications may be in the causal pathway for the association between laboratory measures and CVD risk, we conducted two separate Cox regression analyses: (1) among RA patients not exposed to statins during baseline, censoring them if they initiated statins 
during follow-up; and (2) not adjusting for current use of RA medications.

\section{RESULTS}

There were 44418 eligible RA patients who met the inclusion criteria for the study, and they contributed 46490 episodes of care (figure 1). The mean (SD) age was 49 years and $76 \%$ were women. During baseline, 29\% and 10\% had hypertension and diabetes; $17 \%$ were exposed to statins and $5 \%$ to a non-statin lipid-lowering medication. Detailed data are presented in table 1.

\section{Examination of non-linear association between serum biomarkers and $\mathrm{MI} /$ ischaemic stroke risk}

After adjusting for age and gender, higher CRP and higher ESR levels were associated with increased MI risk and the associations appeared monotonic (figure 2A,B). HDL-cholesterol was linearly and inversely associated with MI risk (figure 2C). We observed a non-linear J-shaped association between LDL-cholesterol and MI risk (figure 2D). To further elucidate the relationship, we examined the association between CRP and $\mathrm{MI}$ in patients whose LDL-cholesterol was $<100 \mathrm{mg} / \mathrm{L}$ $(2.6 \mathrm{mmol} / \mathrm{L})$ and $\geq 100 \mathrm{mg} / \mathrm{L}$. Interestingly, a positive association was only observed among those whose LDL-cholesterol was $\geq 100 \mathrm{mg} / \mathrm{L}(2.6 \mathrm{mmol} / \mathrm{L})$ (data not shown). The results of the spline regression with stroke as the outcome were similar to those observed for MI, and the results are shown in figure 3A-D.

\section{Age-adjusted incidence rates of hospitalised MI and ischaemic stroke}

Higher CRP and ESR levels and lower HDL-cholesterol levels were associated with higher MI incidence. Consistent with the spline, the lowest MI incidence was observed among those with

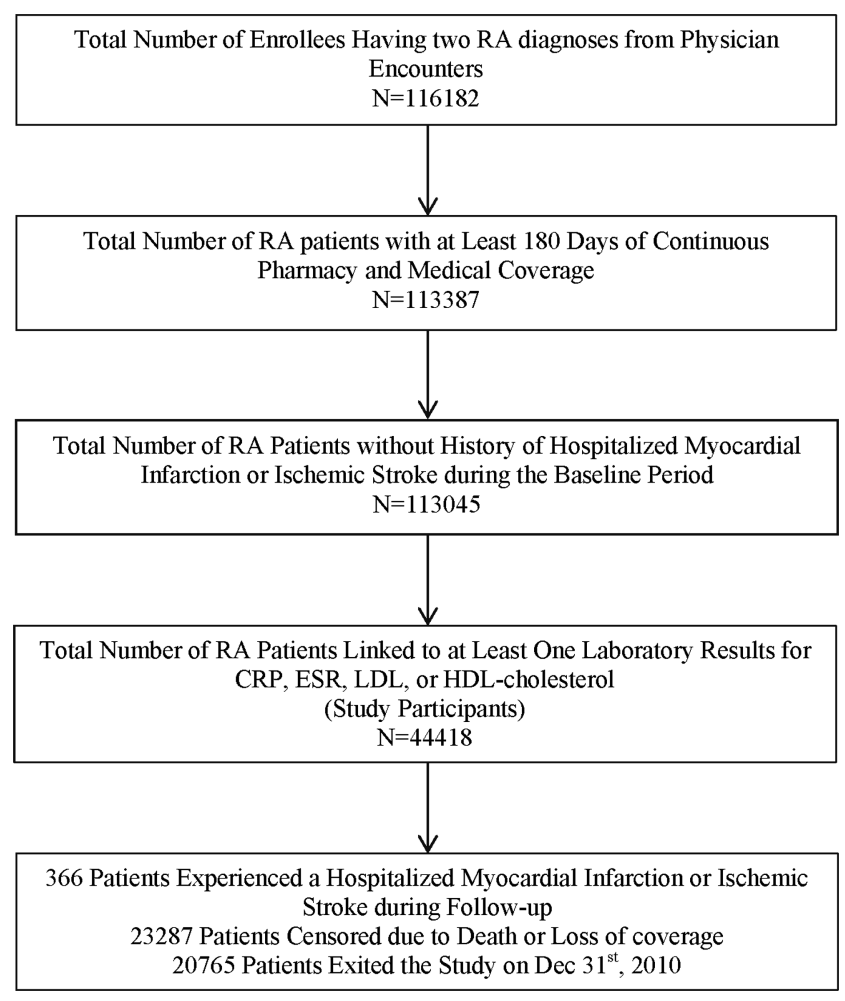

Figure 1 Flow chart of patient selection and outcomes.
Table 1 Patient baseline demographics and clinical characteristics

\begin{tabular}{|c|c|}
\hline Patient baseline characteristics & $N=46,490$ episodes of care \\
\hline Age, Yrs, mean (std) & $49(13)$ \\
\hline Female, $\mathrm{N}, \%$ & $35330(76 \%)$ \\
\hline \multicolumn{2}{|l|}{ Comorbidities } \\
\hline Hypertension, n (\%) & $13283(29 \%)$ \\
\hline Diabetes, $\mathrm{n}(\%)$ & $4529(10 \%)$ \\
\hline Charlson score*, mean (std) & $1.0(0.9)$ \\
\hline \multicolumn{2}{|l|}{ Filled prescription for select medications } \\
\hline Statins, n (\%) & $7952(17 \%)$ \\
\hline Non-statin lipid lowering medications, n (\%) & $2205(5 \%)$ \\
\hline Anti-TNF biologics, n (\%) & $7034(15 \%)$ \\
\hline Non-TNF biologics, n (\%) & $73(0.2 \%)$ \\
\hline Methotrexate, n (\%) & $14337(31 \%)$ \\
\hline Non-methotrexate DMARDs, n (\%) & $10612(23 \%)$ \\
\hline \multicolumn{2}{|c|}{ Average oral prednisone-equivalent glucocorticoid dose, n (\%) } \\
\hline None & $28929(62 \%)$ \\
\hline$>0$ and $\leq 7.5 \mathrm{mg} / \mathrm{d}$ & $15037(32 \%)$ \\
\hline$>7.5$ and $\leq 20 \mathrm{mg} / \mathrm{d}$ & $2318(5 \%)$ \\
\hline$>20 \mathrm{mg} / \mathrm{d}$ & $206(0.4 \%)$ \\
\hline \multicolumn{2}{|l|}{ Distribution of lab values } \\
\hline CRP, mg/L, median (IQR) & $2.9(6.5)$ \\
\hline ESR, mm/hr, median, (IQR) & $8(15)$ \\
\hline LDL, mg/dL [mmol/L], median (IQR) & $108(45)[2.8(1.2)]$ \\
\hline $\mathrm{HDL}$, mg/dL[mmol/L], median (IQR) & $51(20)[1.3(0.5)]$ \\
\hline
\end{tabular}

moderate LDL-cholesterol $(\geq 100 \mathrm{mg} / \mathrm{dL} \quad[2.6 \mathrm{mmol} / \mathrm{L}]$ and $<130 \mathrm{mg} / \mathrm{dL}[3.4 / \mathrm{mmol} / \mathrm{L}])$. MI incidence rates were higher in men than in women, and trends observed among all RA patients were observed among men and women separately (table 2).

Similar to MI, the incidence rates for stroke were higher in men than in women (table 3). Higher CRP, higher ESR and lower HDL-cholesterol levels were associated with higher stroke incidence. The incidence rates were similar among RA patients across all levels of LDL-cholesterol less than $160 \mathrm{mg} / \mathrm{dL}$ $(4.1 \mathrm{mmol} / \mathrm{L})$ and appeared increased among those with LDL-cholesterol $\geq 160 \mathrm{mg} / \mathrm{dL}$. When stratified by gender, except for the inverse association between HDL-cholesterol and stroke risk, opposing trends were observed for men and women.

\section{Multivariable COX-regression analysis}

As shown in table $4, \mathrm{CRP}>10 \mathrm{mg} / \mathrm{L}$ compared with $<1 \mathrm{mg} / \mathrm{L}$ was associated with a twofold increase in MI risk (HR 2.12; 95\% CI 1.02 to 4.38 ). ESR $>42$ compared with $<14$ was associated with a 2.5 -fold increase in MI risk (HR 2.53; 95\% CI 1.48 to 4.31 ). Higher HDL-cholesterol was significantly associated with reduced MI risk (HR $0.37 ; 0.21$ to 0.66). RA patients with moderate LDL-cholesterol between $70 \mathrm{mg} / \mathrm{dL}$ $(1.8 \mathrm{mmol} / \mathrm{L})$ and $130 \mathrm{mg} / \mathrm{dL}(3.4 \mathrm{mmol} / \mathrm{L})$ were at the lowest $\mathrm{MI}$ risk and with LDL-cholesterol $>160 \mathrm{mg} / \mathrm{dL}(4.1 \mathrm{mmol} / \mathrm{L})$ at the highest risk. While somewhat attenuated, similar results were observed for the association between CRP, ESR and HDL-cholesterol with IS.

\section{Sensitivity analysis}

Results from sensitivity analysis in which patients taking statins were excluded are presented in online supplementary 

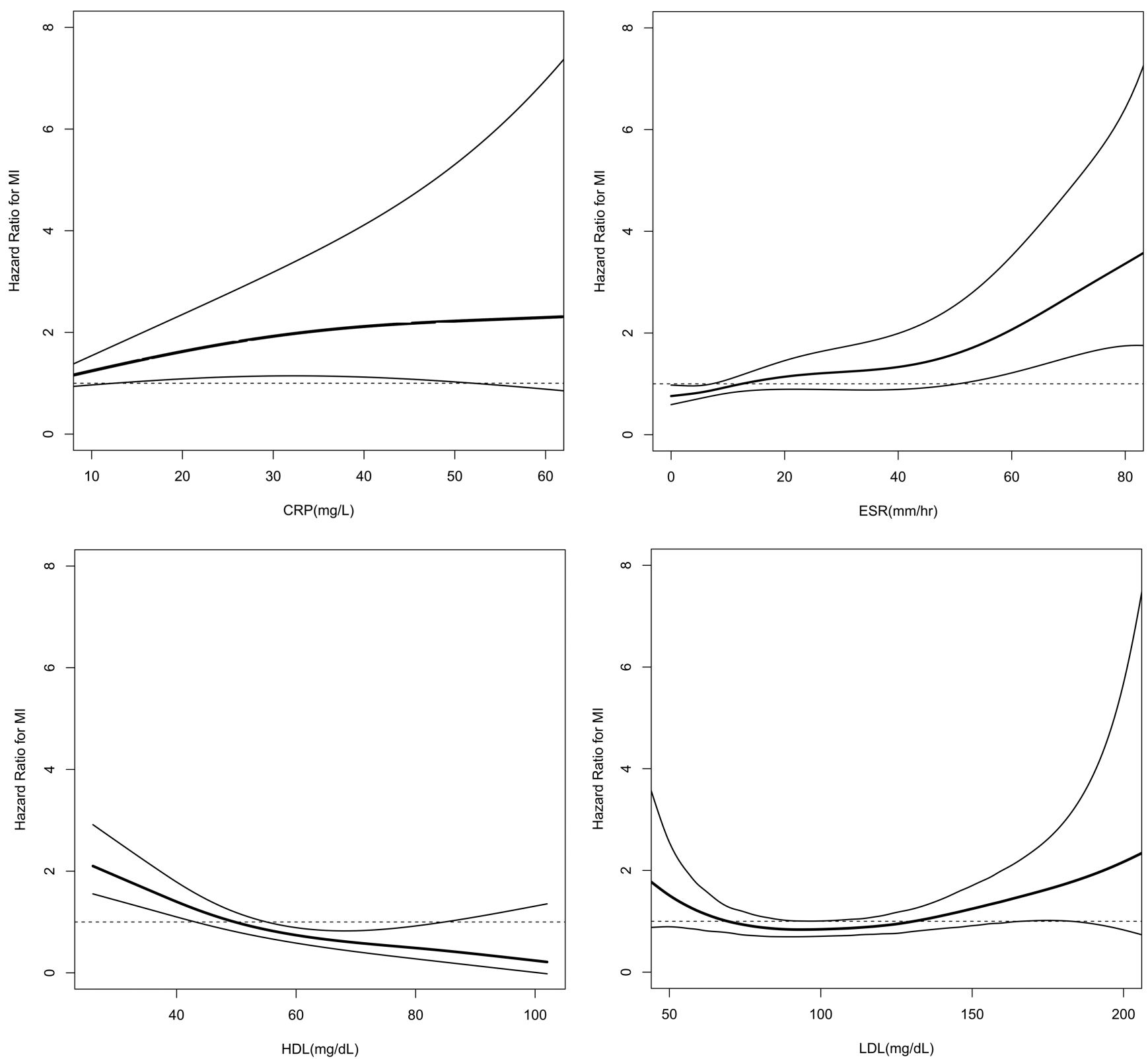

Figure 2 Age and sex-adjusted results from spline regression of the association between serum biomarkers and incident hospitalised myocardial infarction (upper left CRP, upper right erythrocyte sedimentation rate (ESR), lower left high-density lipoprotein-cholesterol and lower right low-density lipoprotein-cholesterol). CRP, C-reactive protein.

appendix A. We observed a significant association between higher ESR and increased MI and stroke risk. While not statistically significant, the dose-response relationship between higher HDL-cholesterol and lower stroke risk we observed in the main analysis was not present. In the sensitivity analysis in which the use of RA medications was not adjusted for, the results were similar to that in the main analysis and details are presented in online supplementary appendix B.

\section{DISCUSSION}

In this retrospective cohort study of RA patients, we observed significant associations between higher CRP, higher ESR and lower HDL-cholesterol levels with increased CVD risk.

The inverse association between HDL-cholesterol and CVD risk in the present study is statistically significant with a clear dose response and is consistently observed across outcomes (both MI and IS) and across all types of analyses (spline, incidence rates, crude and adjusted Cox regression analysis). Together, with similar findings reported in a prior study, ${ }^{17}$ they suggest that high HDL-cholesterol confers a protective effect in RA patients similar to that observed in the general population.

We found a non-linear relationship between LDL-cholesterol and CVD risk with the lowest CVD risk observed among patients with LDL values between $70 \mathrm{mg} / \mathrm{dL}(1.8 \mathrm{mmol} / \mathrm{L})$ and $130 \mathrm{mg} / \mathrm{dL} \quad(3.4 \mathrm{mmol} / \mathrm{L})$. Conflicting findings have been reported regarding the association between dyslipidemia and CVD risk in RA patients. ${ }^{11} 20$ In populations with advanced illnesses, such as heart failure or end-stage renal disease, higher LDL-cholesterol has been paradoxically associated with better survival. $^{32} 33$ The association between low LDL-cholesterol and higher CVD risk may share the same aetiology of the 'reverse epidemiology' of cardiovascular risk factors in such populations. $^{3435}$ 

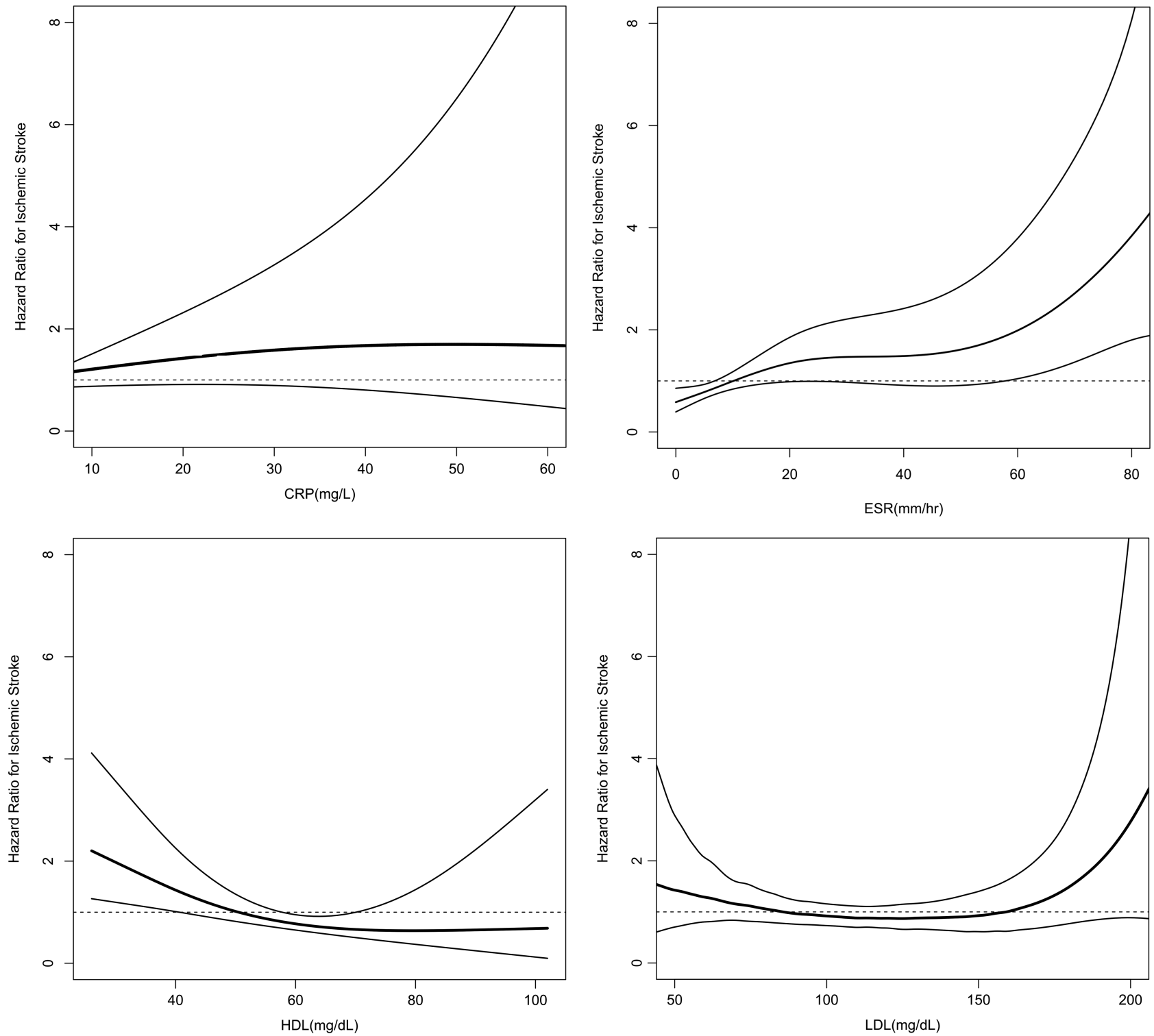

Figure 3 Age and sex-adjusted results from spline regression of the association between serum biomarkers and incident hospitalised ischaemic stroke (upper left CRP, upper right erythrocyte sedimentation rate (ESR), lower left high-density lipoprotein-cholesterol and lower right low-density lipoprotein-cholesterol). CRP, C-reactive protein.

An alternative explanation may be that the relationship between cholesterol and CVD risk is different in RA patients from the general population. In support of this possibility, higher levels of inflammation have been linked to lower cholesterol levels ${ }^{36}$ and data from a randomised trial found increases in total, HDL-cholesterol and LDL-cholesterol levels shortly after treatment initiation among patients with aggressive recent-onset RA. ${ }^{37} \mathrm{~A}$ recent study found that genetic factors contributing to RA susceptibility were associated with reduced LDL-cholesterol level among RA patients. The study created a RA genetic risk score based on 44 published non-HLA RA risk loci and found that each unit increase in the RA risk score was associated with a $0.43 \mathrm{mg} / \mathrm{dL} \quad(0.01 \mathrm{mmol} / \mathrm{L})$ decrease in LDL-cholesterol. $^{38}$

Our finding of a positive and significant association between inflammatory markers and CVD risk is consistent with those reported from prior studies. CRP $>3 \mathrm{mg} / \mathrm{L}$ is positively associated with increased CVD risk in the general population, and our findings suggest that higher CRP continue to confer increased CVD risk above levels seen in the general population. The splines from the present study suggest that the magnitude of the association between CRP and CVD risk was similar for increases up to $30 \mathrm{mg} / \mathrm{L}$ and levelled off afterwards. ESR in the highest quartile was associated with significantly increased CVD risk. These findings underscore the important role played by RA disease-related inflammation in determining the patient's CVD risk and suggests a potential mechanism for potent RA treatment that reduce inflammation to have a beneficial effect on CVD risk.

Because inflammatory markers and use of RA medications could be mutually interdependent, we conducted a sensitivity analysis in which we did not adjust for the use of RA medications. Results were similar to that observed in the main analysis. In addition, we conducted a separate analysis among a homogeneous subgroup of patients not exposed to statins. The exclusion did not alter the findings for MI but resulted in attenuation 
Table 2 Age-adjusted incidence rates for hospitalised MI stratified by levels of inflammatory markers and lipid levels and by gender

\begin{tabular}{|c|c|c|c|c|c|c|c|c|c|}
\hline & \multicolumn{3}{|l|}{ All } & \multicolumn{3}{|l|}{ Female } & \multicolumn{3}{|l|}{ Male } \\
\hline & \# Event & \# Person-years (PY) & $\begin{array}{l}\text { Incidence rate (IR) } \\
\text { per } 1000 \text { PYs }\end{array}$ & \# Event & \# PY & IR per 1000 PYs & \# Event & \# PY & IR per $1000 \mathrm{PYs}$ \\
\hline \multicolumn{10}{|l|}{ CRP (mg/L) } \\
\hline$<1$ & 10 & 8962 & 1.08 & 2 & 6935 & 0.31 & 8 & 2027 & 3.71 \\
\hline $1-2$ & 20 & 12068 & 1.53 & 10 & 9217 & 1.07 & 10 & 2850 & 3.01 \\
\hline $3-10$ & 31 & 8277 & 3.09 & 22 & 6452 & 2.77 & 9 & 1825 & 4.42 \\
\hline$\geq 10$ & 36 & 13483 & 2.40 & 17 & 10690 & 1.43 & 19 & 2794 & 6.24 \\
\hline \multicolumn{10}{|l|}{$\mathrm{ESR}(\mathrm{mm} / \mathrm{h})$} \\
\hline$<14$ & 53 & 31757 & 1.54 & 24 & 23479 & 0.96 & 29 & 8279 & 3.22 \\
\hline $14-27$ & 24 & 9156 & 2.26 & 12 & 7647 & 1.34 & 12 & 1509 & 7.19 \\
\hline $28-42$ & 10 & 4749 & 2.09 & 5 & 4012 & 1.28 & 5 & 736 & 6.68 \\
\hline$>42$ & 20 & 3890 & 4.41 & 15 & 3184 & 3.80 & 5 & 706 & 7.79 \\
\hline \multicolumn{10}{|c|}{ HDL-cholesterol (mg/dL/mmol/L) } \\
\hline$<40 / 1.0$ & 52 & 9191 & 4.38 & 14 & 4748 & 1.50 & 38 & 4443 & 7.07 \\
\hline $40 / 1.0-49 / 1.3$ & 35 & 9949 & 3.94 & 16 & 6946 & 2.55 & 19 & 3003 & 6.80 \\
\hline $50 / 1.3-59 / 1.5$ & 19 & 10508 & 1.85 & 12 & 8551 & 1.58 & 7 & 1958 & 2.89 \\
\hline$\geq 60 / 1.6$ & 18 & 11824 & 1.36 & 14 & 10861 & 1.09 & 4 & 963 & 4.24 \\
\hline \multicolumn{10}{|c|}{ LDL-cholesterol (mg/dL/mmol/L) } \\
\hline$<100 / 2.6$ & 52 & 17015 & 2.75 & 22 & 12458 & 1.60 & 30 & 4558 & 6.09 \\
\hline 100/2.6-129/3.3 & 34 & 14009 & 2.01 & 15 & 10780 & 1.21 & 19 & 3230 & 4.70 \\
\hline 130/3.4-159/4.1 & 24 & 7393 & 2.73 & 12 & 5586 & 1.89 & 12 & 1807 & 5.31 \\
\hline$>160 / 4.1$ & 11 & 2775 & 3.65 & 5 & 2140 & 2.12 & 6 & 635 & 8.75 \\
\hline
\end{tabular}

CRP, C-reactive protein; ESR, erythrocyte sedimentation rate; HDL, high-density lipoprotein; LDL, low-density lipoprotein.

of the associations between HDL-cholesterol and LDL-cholesterol with stroke. However, interpretation of this subgroup analysis is difficult due to the small number of stroke events, especially among those with higher LDL-cholesterol levels.
Despite large numbers of RA patients, we still observed relatively small numbers of MI and IS. This made some findings difficult to interpret after age and sex stratification. Another limitation of the study was the lack of information on important clinical CVD risk factors such as smoking or blood pressure

Table 3 Age-adjusted incidence rates for hospitalised ischaemic stroke stratified by levels of inflammatory markers and lipid levels and by gender

\begin{tabular}{|c|c|c|c|c|c|c|c|c|c|}
\hline & \multicolumn{3}{|l|}{ All } & \multicolumn{3}{|l|}{ Female } & \multicolumn{3}{|l|}{ Male } \\
\hline & \# Event & \# Person-years (PY) & $\begin{array}{l}\text { Incidence rate (IR) } \\
\text { per } 1000 \text { PYs }\end{array}$ & \# Event & \# PY & IR per $1000 \mathrm{PYs}$ & \# Event & \# PY & IR per 1000 PYs \\
\hline \multicolumn{10}{|l|}{ CRP (mg/L) } \\
\hline$<1$ & 8 & 8971 & 0.84 & 3 & 6937 & 0.42 & 5 & 2034 & 2.29 \\
\hline $1-2$ & 13 & 12104 & 0.95 & 9 & 9213 & 0.92 & 4 & 2890 & 1.03 \\
\hline $3-10$ & 18 & 8289 & 1.80 & 11 & 6457 & 1.40 & 7 & 1832 & 3.32 \\
\hline$\geq 10$ & 21 & 13511 & 1.46 & 12 & 10699 & 1.15 & 9 & 2812 & 2.62 \\
\hline \multicolumn{10}{|l|}{$\operatorname{ESR}(\mathrm{mm} / \mathrm{h})$} \\
\hline$<14$ & 34 & 31819 & 0.98 & 19 & 23481 & 0.75 & 15 & 8338 & 1.67 \\
\hline $14-27$ & 17 & 9172 & 1.75 & 14 & 7658 & 1.80 & 3 & 1514 & 1.52 \\
\hline $28-42$ & 8 & 4762 & 1.65 & 3 & 4021 & 0.66 & 5 & 741 & 7.22 \\
\hline$>42$ & 14 & 3889 & 3.03 & 13 & 3186 & 3.37 & 1 & 702 & 1.75 \\
\hline \multicolumn{10}{|c|}{ HDL-cholesterol, $\mathrm{mg} / \mathrm{dL}[\mathrm{mmol} / \mathrm{L}]$} \\
\hline$<40 / 1.0$ & 27 & 9283 & 2.62 & 13 & 4753 & 2.36 & 14 & 4530 & 2.86 \\
\hline $40 / 1.0-49 / 1.3$ & 20 & 9997 & 1.81 & 10 & 6968 & 1.06 & 10 & 3028 & 3.31 \\
\hline $50 / 1.3-59 / 1.5$ & 14 & 10540 & 1.62 & 8 & 8559 & 1.40 & 6 & 1981 & 2.54 \\
\hline$\geq 60 / 1.6$ & 12 & 11839 & 0.98 & 10 & 10868 & 0.93 & 2 & 970 & 1.66 \\
\hline \multicolumn{10}{|c|}{ LDL-cholesterol mg/dL [mmol/L] } \\
\hline$<100 / 2.6$ & 31 & 17147 & 1.64 & 14 & 12481 & 1.04 & 17 & 4666 & 3.34 \\
\hline $100 / 2.6-129 / 3.3$ & 22 & 14055 & 1.43 & 13 & 10796 & 1.12 & 9 & 3259 & 2.50 \\
\hline $130 / 3.4-159 / 4.1$ & 12 & 7404 & 1.56 & 8 & 5592 & 1.34 & 4 & 1812 & 2.28 \\
\hline$>160 / 4.1$ & 7 & 2780 & 2.51 & 6 & 2137 & 2.76 & 1 & 643 & 1.76 \\
\hline
\end{tabular}

CRP, C-reactive protein; ESR, erythrocyte sedimentation rate; HDL, high-density lipoprotein; LDL, low-density lipoprotein; MI, myocardial infarction. 
Table 4 Adjusted HRs* of hospitalised myocardial infarction and ischaemic stroke among patients with rheumatoid arthritis (eight separate models)

\begin{tabular}{|c|c|c|}
\hline & MI & Stroke \\
\hline \multicolumn{3}{|l|}{ CRP (mg/L) } \\
\hline$<1$ & Reference & Reference \\
\hline $1-2$ & 1.19 (0.56 to 2.55$)$ & 1.03 (0.43 to 2.46$)$ \\
\hline $3-10$ & 1.72 (0.85 to 3.48$)$ & 1.37 (0.62 to 3.06$)$ \\
\hline$>10$ & 2.12 (1.02 to 4.38$)$ & 1.76 (0.77 to 4.03$)$ \\
\hline \multicolumn{3}{|l|}{ ESR $(\mathrm{mm} / \mathrm{h})$} \\
\hline$<14$ & Reference & Reference \\
\hline $14-27$ & $1.48(0.90$ to 2.45$)$ & 1.46 (0.81 to 2.62$)$ \\
\hline $28-42$ & 1.20 (0.59 to 2.43$)$ & 1.29 (0.58 to 2.86$)$ \\
\hline$>42$ & 2.53 (1.48 to 4.31$)$ & 2.51 (1.33 to 4.75$)$ \\
\hline \multicolumn{3}{|c|}{ HDL-cholesterol (mg/dL/mmol/L) } \\
\hline$<40 / 1.0$ & Reference & Reference \\
\hline 40/1.0-49/1.3 & $0.68(0.44$ to 1.05$)$ & 0.78 (0.43 to 1.42 ) \\
\hline 50/1.3-59/1.5 & $0.40(0.24$ to 0.70$)$ & 0.57 (0.28 to 1.15$)$ \\
\hline$\geq 60 / 1.6$ & $0.37(0.21$ to 0.66$)$ & $0.48(0.22$ to 1.01$)$ \\
\hline \multicolumn{3}{|c|}{ LDL-cholesterol (mg/dL/mmol/L) } \\
\hline$<70 / 1.8$ & Reference & Reference \\
\hline 70/1.8-99/2.6 & 0.53 (0.31 to 0.92$)$ & 1.15 (0.50 to 2.65$)$ \\
\hline $100 / 2.6-129 / 3.3$ & $0.63(0.37$ to 1.10$)$ & 1.16 (0.49 to 2.80$)$ \\
\hline 130/3.4-160/4.1 & $0.88(0.48$ to 1.60$)$ & 1.26 (0.48 to 3.26$)$ \\
\hline$>160 / 4.1$ & $1.12(0.54$ to 2.31$)$ & 1.94 (0.66 to 5.75$)$ \\
\hline
\end{tabular}

levels or on other important influences on CVD risk, such as poverty. A 180-day baseline period may not be sufficiently long enough to capture comorbidities, CVD risk factors and history of CVD events. Because these factors might be associated with inflammation, RA severity or CVD risk, they may be sources of unmeasured or residual confounding. Another limitation of the study is that we cannot guarantee that the lipids were measured in a fasting state. However, a recent study showed that HDL-cholesterol levels by fasting time (from 1 to $16 \mathrm{~h}$ fasting time) varied by less than $2 \%$ and LDL-cholesterol by less than $10 \% .^{39}$

Despite these limitations, our findings underline the importance of systemic inflammation in determining the CVD risk of RA patients and provide additional evidence supporting a complicated relationship between dyslipidemia and CVD risk among these patients. Our findings also highlight the need to correctly classify RA patients in terms of their cardiovascular risk. This is relevant to the European Society of Cardiology and the European Atherosclerosis Society (ESC/EAS) guidelines that set treatment goals on the basis of estimated cardiovascular risk. ${ }^{40}$ The recently issued new American College of Cardiology-American Heart Association (ACC-AHA) guidelines represent a shift from LDL-cholesterol goals to greater reliance on level of risk. ${ }^{41}$ Given the renewed focus on risk and significantly elevated CVD risk among RA patients, the development and refinement of cardiovascular risk calculation in population with chronic inflammation should be regarded a high-priority area for future research.

Contributors All authors made substantial contributions to conception and design, data acquisition/analysis, interpretation, drafting or critical revision of manuscript and approved the manuscript for publication. They have met the International Committee of Medical Journal Editors Recommendations for the Conduct, Reporting, Editing, and Publication of Scholarly Work in Medical Journals (ICMJE Recommendations 2013) recommend criteria for authorship.

Funding Funding for this study was supported by the Agency for Healthcare Research and Quality (R01 HS018517) and Genentech. The design, analysis and interpretation of the study were solely guided by the authors.

Competing interests JRC has received research grants and consulting fees from Amgen, Abbott, BMS, Pfizer, Eli Lilly, Janssen, UCB, Roche/Genentech and CORRONA. MMS has received research grants from Amgen, Pfizer and Peers for Progress, a collaboration between the American Family Physicians Foundation and the Eli Lilly Foundation. ED has received research grants from Amgen.

\section{Patient consent Obtained.}

Ethics approval IRB.

Provenance and peer review Not commissioned; externally peer reviewed.

\section{REFERENCES}

1 Solomon DH, Goodson NJ, Katz JN, et al. Patterns of cardiovascular risk in rheumatoid arthritis. Ann Rheum Dis 2006;65:1608-12.

2 Solomon DH, Karlson EW, Rimm EB, et al. Cardiovascular morbidity and mortality in women diagnosed with rheumatoid arthritis. Circulation 2003;107:1303-7.

3 Maradit-Kremers H, Crowson CS, Nicola PJ, et al. Increased unrecognized coronary heart disease and sudden deaths in rheumatoid arthritis: a population-based cohort study. Arthritis Rheum 2005;52:402-11.

4 Kremers HM, Crowson CS, Therneau TM, et al. High ten-year risk of cardiovascular disease in newly diagnosed rheumatoid arthritis patients: a population-based cohort study. Arthritis Rheum 2008;58:2268-74.

5 Avina-Zubieta JA, Choi HK, Sadatsafavi M, et al. Risk of cardiovascular mortality in patients with rheumatoid arthritis: a meta-analysis of observational studies. Arthritis Rheum 2008:59:1690-7.

6 Crowson CS, Nicola PJ, Kremers HM, et al. How much of the increased incidence of heart failure in rheumatoid arthritis is attributable to traditional cardiovascular risk factors and ischemic heart disease? Arthritis Rheum 2005;52:3039-44.

7 Gonzalez A, Maradit Kremers H, Crowson CS, et al. Do cardiovascular risk factors confer the same risk for cardiovascular outcomes in rheumatoid arthritis patients as in non-rheumatoid arthritis patients? Ann Rheum Dis 2008;67:64-9.

8 Crowson CS, Matteson EL, Roger VL, et al. Usefulness of risk scores to estimate the risk of cardiovascular disease in patients with rheumatoid arthritis. Am J Cardiol 2012:110:420-4.

9 Corrales A, Gonzalez-Juanatey C, Peiro ME, et al. Carotid ultrasound is useful for the cardiovascular risk stratification of patients with rheumatoid arthritis: results of a population-based study. Ann Rheum Dis 2014;73:722-7.

10 Myasoedova E, Gabriel SE. Cardiovascular disease in rheumatoid arthritis: a step forward. Curr Opin Rheumatol 2010;22:342-7.

11 Myasoedova E, Crowson CS, Kremers HM, et al. Lipid paradox in rheumatoid arthritis: the impact of serum lipid measures and systemic inflammation on the risk of cardiovascular disease. Ann Rheum Dis 2011;70:482-7.

12 Solomon DH, Kremer J, Curtis JR, et al. Explaining the cardiovascular risk associated with rheumatoid arthritis: traditional risk factors versus markers of rheumatoid arthritis severity. Ann Rheum Dis 2010;69:1920-5.

13 Banerjee S, Compton AP, Hooker RS, et al. Cardiovascular outcomes in male veterans with rheumatoid arthritis. Am J Cardiol 2008;101:1201-5.

14 Graf J, Scherzer R, Grunfeld C, et al. Levels of C-reactive protein associated with high and very high cardiovascular risk are prevalent in patients with rheumatoid arthritis. PLOS ONE 2009;4:e6242.

15 Gonzalez-Gay MA, Gonzalez-Juanatey C, Pineiro A, et al. High-grade C-reactive protein elevation correlates with accelerated atherogenesis in patients with rheumatoid arthritis. J Rheumatol 2005:32:1219-23.

16 Gonzalez-Gay MA, Gonzalez-Juanatey C, Lopez-Diaz MJ, et al. HLA-DRB1 and persistent chronic inflammation contribute to cardiovascular events and cardiovascular mortality in patients with rheumatoid arthritis. Arthritis Rheum 2007:57:125-32.

17 Innala L, Moller B, Ljung L, et al. Cardiovascular events in early RA are a result of inflammatory burden and traditional risk factors: a five year prospective study. Arthritis Res Ther 2011;13:R131.

18 Gabriel SE, Crowson CS. Risk factors for cardiovascular disease in rheumatoid arthritis. Curr Opin Rheumatol 2012;24:171-6.

19 van Halm VP, Nielen MM, Nurmohamed MT, et al. Lipids and inflammation: serial measurements of the lipid profile of blood donors who later developed rheumatoid arthritis. Ann Rheum Dis 2007:66:184-8.

20 Semb AG, Kvien TK, Aastveit AH, et al. Lipids, myocardial infarction and ischaemic stroke in patients with rheumatoid arthritis in the Apolipoprotein-related Mortality RISk (AMORIS) Study. Ann Rheum Dis 2010;69:1996-2001.

21 Myasoedova E, Crowson CS, Kremers HM, et al. Total cholesterol and LDL levels decrease before rheumatoid arthritis. Ann Rheum Dis 2010:69:1310-4. 
22 Roumie CL, Mitchel E, Gideon PS, et al. Validation of ICD-9 codes with a high positive predictive value for incident strokes resulting in hospitalization using Medicaid health data. Pharmacoepidemiol Drug Saf 2008;17:20-6.

23 Tirschwell DL, Longstreth WT Jr. Validating administrative data in stroke research. Stroke 2002;33:2465-70.

24 Cutrona SL, Toh S, lyer A, et al. Validation of acute myocardial infarction in the Food and Drug Administration's Mini-Sentinel program. Pharmacoepidemiol Drug Saf 2013;22:40-54.

25 Kiyota Y, Schneeweiss S, Glynn RJ, et al. Accuracy of Medicare claims-based diagnosis of acute myocardial infarction: estimating positive predictive value on the basis of review of hospital records. Am Heart J 2004;148:99-104.

26 Metcalfe A, Neudam A, Forde $S$, et al. Case definitions for acute myocardial infarction in administrative databases and their impact on in-hospital mortality rates. Health Serv Res 2013:48:290-318.

27 de Groot V, Beckerman H, Lankhorst GJ, et al. How to measure comorbidity. a critical review of available methods. J Clin Epidemiol 2003:56:221-9.

28 Eilers PHC, Marx BD. Flexible smoothing with B-splines and penalties. Stat SCi 1996;11:89-102

29 National Cholesterol Education Program (NCEP) Expert Panel on Detection, Evaluation, and Treatment of High Blood Cholesterol in Adults (Adult Treatment Panel III). Third Report of the National Cholesterol Education Program (NCEP) Expert Panel on Detection, Evaluation, and Treatment of High Blood Cholesterol in Adults (Adult Treatment Panel III) final report. Circulation 2002;106:3143-421.

30 McCarey DW, McInnes IB, Madhok R, et al. Trial of Atorvastatin in Rheumatoid Arthritis (TARA): double-blind, randomised placebo-controlled trial. Lancet 2004;363:2015-21.

31 Lin DY, Wei LJ. The robust inference for the Cox proportional hazards model. J Am Stat Assoc 1989:84:1074-8.

32 Velavan $\mathrm{P}$, Huan Loh $\mathrm{P}$, Clark A, et al. The cholesterol paradox in heart failure. Congest Heart Fail 2007;13:336-41.
33 Kovesdy CP, Anderson JE, Kalantar-Zadeh K. Inverse association between lipid levels and mortality in men with chronic kidney disease who are not yet on dialysis: effects of case mix and the malnutrition-inflammation-cachexia syndrome. J Am Soc Nephrol 2007; 18:304-11.

34 Kalantar-Zadeh K, Block G, Horwich T, et al. Reverse epidemiology of conventional cardiovascular risk factors in patients with chronic heart failure. J Am Coll Cardiol 2004:43:1439-44.

35 Guder G, Frantz S, Bauersachs J, et al. Reverse epidemiology in systolic and nonsystolic heart failure: cumulative prognostic benefit of classical cardiovascular risk factors. Circ Heart Fail 2009:2:563-71.

36 Choy E, Sattar N. Interpreting lipid levels in the context of high-grade inflammatory states with a focus on rheumatoid arthritis: a challenge to conventional cardiovascular risk actions. Ann Rheum Dis 2009;68:460-9.

37 Navarro-Millan I, Charles-Schoeman C, Yang S, et al. Changes in lipoproteins associated with methotrexate or combination therapy in early rheumatoid arthritis: results from the treatment of early rheumatoid arthritis trial. Arthritis Rheum 2013:65:1430-8

38 Liao KP, Diogo D, Cui J, et al. Association between low density lipoprotein and rheumatoid arthritis genetic factors with low density lipoprotein levels in rheumatoid arthritis and non-rheumatoid arthritis controls. Ann Rheum Dis 2014; 73:1170-5

39 Sidhu D, Naugler C. Fasting time and lipid levels in a community-based population: a cross-sectional study. Arch Intern Med 2012;172:1707-10.

40 Catapano AL, Reiner Z, De Backer G, et al. ESC/EAS Guidelines for the management of dyslipidaemias The Task Force for the management of dyslipidaemias of the European Society of Cardiology (ESC) and the European Atherosclerosis Society (EAS). Atherosclerosis 2011;217:3-46.

41 Psaty BM, Weiss NS. 2013 ACC/AHA Guideline on the treatment of blood cholesterol: a fresh interpretation of old evidence. JAMA 2014;311: $461-2$ 